\title{
What is infidelity? Perceptions based on biological sex and personality
}

This article was published in the following Dove Press journal:

Psychology Research and Behavior Management

12 May 2011

Number of times this article has been viewed

\author{
Victoria Thornton \\ Alexander Nagurney \\ Texas State University - San Marcos, \\ San Marcos, Texas, USA
}

\begin{abstract}
The study examines perceptions of infidelity, paying particular attention to how these perceptions differ based on biological sex and personality traits, specifically agency and communion and their unmitigated counterparts. The study utilizes a sample of 125 male and 233 female college students. In addition to the personality measures, participants completed a 19-item checklist that assessed their perceptions of specific items that could potentially be construed as infidelity. It was hypothesized that females would construe more items as infidelity than would males. It was also predicted that unmitigated communion and communion would be positively correlated with these perceptions and that unmitigated agency would be negatively correlated with these perceptions. No correlation was predicted between agency and infidelity. All hypotheses were supported. Implications and suggestions for future research are discussed. Keywords: infidelity, communion, agency, questionnaire, relationship
\end{abstract}

\section{What is infidelity?}

Experiencing an act of what one considers to be infidelity within the confines of a committed relationship is without argument a personal and often traumatic event. But what constitutes such an act? The purpose of the current investigation is to begin exploring this question. Infidelity has been defined in a multitude of ways and comprises a number of activities, including "having an affair, extramarital relationship, cheating, sexual intercourse, oral sex, kissing, fondling, emotional connections that are beyond friendships, friendships, internet relationships, and pornography use, among others, and has been defined as involvement in romantic relationships outside of one's active committed relationship which result in a sense of relational betrayal". ${ }^{1}$ Alfred Kinsey was among the first social scientists to examine perceptions of infidelity. He distinguished between two types: emotional and sexual. ${ }^{2}$ Sexual infidelity is considered to be engaging in sexual intercourse with someone other than one's partner, whereas emotional infidelity is considered to be "falling in love" or sharing a deep emotional bond with someone other than one's partner. ${ }^{3}$ Clearly, there is no universal operational definition of infidelity, and Blow and Harnett ${ }^{4}$ have shown that there are no agreeable or simple guidelines for defining infidelity or those behaviors that fall within this category.

Many people think infidelity or "cheating" implies a sexual or physical act with someone other than their partner, but that is not always the case. Recent research has shown a particularly prominent difference between how men and women respond to different types of perceived infidelity. ${ }^{5}$ This research has demonstrated that women consider an intense emotional relationship outside of their own as an unfaithful involvement, even when there is no physical component. Men, on the other hand,
Correspondence:Alexander Nagurney Department of Psychology, 60I University Drive, San Marcos, TX 78666, USA

Tel + I 5122453165

Fax +15122453153

Email an18@txstate.edu 
consider primarily physical contact, typically sexual, to constitute infidelity, much more so than an emotional involvement outside of their relationship. ${ }^{6}$ Ultimately, infidelity might be considered to be feelings or behaviors that go against a partner's expectations for the exclusivity of the relationship.

The present study was conducted in an effort to better define how people perceive infidelity by using a broader operational definition. Based on literature reviewed and for our purposes in this study, we classify infidelity, in general, as a breach of trust, either emotional or physical, that signifies a potential lack of commitment to one's romantic partner. The study seeks to showcase those items, or acts, that people commonly define as infidelity using a multiple-item checklist. This investigation hypothesized that women will check off overall more items as constituting what they perceive to be infidelity than will men.

\section{Gender-related traits}

Gender-related traits are those traits that are considered to be more common in one sex than in the other. There are two main types of gender-related traits, agency and communion. ${ }^{7}$ Agency is the extent to which one focuses on personal achievements and believes that one exists as an individual. It emphasizes such issues as self-protection and the creation of a unique identity. Communion, on the other hand, is the extent to which one focuses on establishing and maintaining supportive relationships and the belief that one is part of a larger social structure. It emphasizes such issues as cooperation and attachment. ${ }^{8}$ It should be noted that each of these traits, in moderation, contributes to an overall sense of well-being. Most individuals possess both traits to a moderate extent and can therefore garner the benefits of each. However, there are instances when an individual can possess one of these traits to the exclusion of the other. The traits then become known as unmitigated agency and unmitigated communion, and those unmitigated versions of the gender-related traits are associated with less socially acceptable outcomes. In other words, the unmitigated versions of both agency and communion tend to be those traits that are considered more harmful and tend to have negative effects on one's psychological and physiological well-being. ${ }^{7,8}$

The unmitigated agency individual tends to focus on their own achievements and goals to the exclusion of others' needs and tends to be generally more avoidant. These individuals will therefore be more socially isolated and will lack the supportive networks that could help them in times of stress. The unmitigated communion individual tends to be more focused on the needs of others to the point where their own needs are largely ignored. These individuals thus will generally have a lesser sense of their own identity. Research has shown that females tend to score higher than males on measures of communion and unmitigated communion, whereas males tend to score higher than females on measures of agency and unmitigated agency. ${ }^{7}$ It has also been shown that although agency and communion are positively related to selfesteem and well-being, unmitigated agency and unmitigated communion are positively related to anxiety and depression. ${ }^{9}$ In keeping with past research, the current study assessed individuals on these traits and hypothesized that females would score higher on measures of communion and unmitigated communion and that males would score higher on measures of agency and unmitigated agency.

\section{Infidelity and gender-related traits}

Now that both perceived infidelity and those gender-related traits that apply to the current study are defined, the issue of how infidelity and the gender-related traits might potentially be associated with each other will be explored. Previous research correlates unmitigated communion with traits such as low selfesteem and a more co-dependent nature and with being overly concerned or obsessive with the state of a relationship or with the partner involved. ${ }^{9}$ Because of this pattern of associations, we hypothesized that individuals scoring high in unmitigated communion will endorse more items on the perceived infidelity questionnaire, possibly as a result of perceiving more items as a threat to their relationship. Therefore, a positive correlation between unmitigated communion and perceived infidelity is predicted. On the contrary, unmitigated agency is correlated with traits such as hostility, greed, and arrogance and with being overly concerned with oneself. ${ }^{9}$ Because of these associations, we hypothesized that the individuals scoring high in unmitigated agency will endorse fewer items on the infidelity questionnaire, possibly as a result of their lack of concern for relationships. Therefore, a negative correlation between unmitigated agency and perceived infidelity was predicted.

We next hypothesized about agency and communion, the two more socially healthy gender-related traits. Based on the characteristics of agency and its correlation with achievement, power, and competence, ${ }^{8}$ we hypothesized that there would be no correlation between agency and perceived infidelity, as a result of the emotional security of individuals scoring high in agency.

Because of communion and its focus on relationships, we were unsure of the impact it would have on the results 
of the study. On one hand, it is possible that communion would be negatively correlated with infidelity, as a result of the communion individual possessing a more positive outlook on relationships and therefore feeling less threatened or less negatively about more items on the questionnaire. They would therefore have fewer perceptions of infidelity. It is also possible, on the other hand, that communion would be positively correlated with infidelity, as a result of those scoring high on communion being potentially overinvolved or overindulgent in their relationships, akin to a "priming effect" of communion, setting people up to see infidelity in others and their actions. As a result, no firm hypothesis was set forward concerning how communion would be associated with infidelity perceptions.

\section{Methods}

\section{Participants}

A total of 361 undergraduate college students recruited from a large survey course completed all measures of the study. The participants were given extra credit in return for their time. In the event that a student did not want to participate or did not feel comfortable responding to the questionnaires in the study, they were given the opportunity to complete another assignment (ie, a summary of a short journal article) for an equal amount of extra credit. Participants ranged in age from 18 to 37 years (mean 20.39 years) and identified themselves as Caucasian (58.2\%), Hispanic (27.4\%), Black (8\%), or Other $(6.4 \%)$. There were 233 female participants $(65 \%)$ and 125 male participants (35\%). A total of 133 participants identified themselves as "single and not dating" (36.8\%), 111 as "casually dating" (30.7\%), and 117 as "married/in a committed relationship" (32.4\%). Participants were predominantly heterosexual (94.5\%), with homosexual (3.3\%), bisexual $(1.4 \%)$, and other $(0.6 \%)$ encompassing the rest.

\section{Procedure}

Participants were asked to respond to three questionnaires, one involving perceptions of infidelity, one involving ratings of agency, communion, and unmitigated agency, and one for ratings of unmitigated communion. For the perceived infidelity questionnaire, participants were asked to check off those situations they considered to constitute infidelity. For both of the gender-related trait surveys, Likert scales were used ranging from 1 to 5 on how strongly they agreed or disagreed with the statement.

It should be noted that, prior to the collection of any data, approval was obtained from the university's Institutional Review Board following an expedited review by the board.
Participant confidentiality was maintained through the use of identification numbers rather than names when collecting and analyzing data. Participants signed an informed consent form that was submitted separately from their responses to the questionnaires.

\section{Instruments}

\section{Infidelity questionnaire}

The infidelity questionnaire was constructed in a checklist format and consisted of 19 statements. Participants were required to determine whether or not they considered each statement to be an act of infidelity. The questionnaire was created specifically for this study $(\alpha=0.76)$. Examples of the statements are "being in a committed, monogamous relationship while hiding a physical attachment to someone other than your partner", "an intimate kiss (kiss on the lips) with someone other than your partner", "fantasizing about someone other than your partner", "engaging in sexual intercourse with someone other than your partner", "sharing an intimate emotional bond with someone other than your partner", "general dishonesty (lying about anything to your partner)", "flirting with someone other than your partner", "oral sex with someone other than your partner", "sexting (the act of sending sexually explicit messages or photos electronically, usually between cell phones)", and "any breach of the trust in the relationship". Given that no questionnaire used to assess these ideas could be located, the authors wrote the items from scratch as a starting point for exploring perceptions of infidelity. See Appendix A for a full list of the items.

\section{Gender-related traits}

The participants also completed the extended version of the Personal Attributes Questionnaire, the measure used to assess the traits of agency, unmitigated agency, and communion ${ }^{10,11}$ Each subscale consisted of eight items, which were rated on a 5-point scale ranging from 1 (not at all identifying with the trait) to 5 (very much identifying with the trait). Agency items reflected a positive orientation toward the self, such as "independent", "self-confident", and "never gives up". Unmitigated agency items reflected a negative orientation toward the self, exclusive of others, such as self-absorption (eg, arrogance and greed) and a negative view of others (eg, hostile and cynical). Communion items reflected a positive other orientation such as "helpful", "aware of others feelings", and "understanding of others". These scales have high internal consistencies and well-established reliability and validity. ${ }^{7,9}$ (Spence et al, 1979; Spence and Helmreich, 
1978). In the present study, the internal consistencies for the scales were comparable with previous research: agency (0.76), communion (0.76), and unmitigated agency (0.72).

Unmitigated communion was assessed with the Revised Unmitigated Communion Scale. ${ }^{12}$ Participants indicated once again their agreement with items on a 5-point scale. The scale applies to those who place others' needs before their own and distress over concern for others. Examples of the items included "I always place the needs of others above my own", "I can't say no when someone asks me for help", and "I often worry about other people's problems". Previous research has shown that this scale demonstrates acceptable internal consistency, ranging from 0.7 to 0.8 and high test-retest reliability. ${ }^{9,12,13}$ In the present study, the internal consistency was $\alpha=0.74$.

\section{Results}

\section{Main hypotheses}

The first hypothesis of the study was that females would consider more items on the checklist to constitute infidelity than would males. This hypothesis was confirmed with a $t$-test, $t(357)=-5.53, P<0.001$. Females checked off an average of 10.13 items (standard deviation $[\mathrm{SD}]=3.31$ ), whereas males checked off an average of 8.16 items $(\mathrm{SD}=3.05)$.

The second set of hypotheses stated that females would score higher on measures of communion and unmitigated communion, whereas males would score higher than females on measures of agency and unmitigated agency. These predictions were confirmed in all cases. A multivariate analysis of variances revealed significant differences for communion, $F(1,355)=30.33, P<0.001$, for unmitigated communion, $F(1,355)=16.16, P<0.001$, for agency, $F(1,355)=8.00, P<0.001$, and for unmitigated agency, $F(1,355)=12.51, P<0.001$. All means were in the expected directions. See Table 1 for a summary of the means separated by sex.

The final set of hypotheses deals with the relationships of the gender-related traits to perceptions of infidelity. It was predicted that unmitigated communion would be positively associated with the number of items on the checklist con-

Table I Means and standard deviations separated by sex

\begin{tabular}{lllll}
\hline Variable & Male & Female & $\boldsymbol{t}$ value & $\boldsymbol{P}$ value \\
\hline Infidelity perceptions & $8.16(3.05)$ & $10.13(3.3 \mathrm{I})$ & -5.53 & $<0.00 \mathrm{I}$ \\
Communion & $3.72(0.55)$ & $4.05(0.54)$ & -5.47 & $<0.00 \mathrm{I}$ \\
Unmitigated communion & $3.05(0.65)$ & $3.32(0.6 \mathrm{I})$ & -3.97 & $<0.00 \mathrm{I}$ \\
Agency & $3.66(0.62)$ & $3.46(0.6 \mathrm{I})$ & 2.85 & 0.005 \\
Unmitigated agency & $2.57(0.53)$ & $2.36(0.54)$ & 3.54 & $<0.00 \mathrm{I}$ \\
\hline
\end{tabular}

sidered to constitute infidelity, and this prediction was supported. Unmitigated communion's correlation with the number of items checked on the infidelity questionnaire was 0.17 ( $P<0.001)$. Communion's correlation with the number of items checked was $0.21(P<0.001)$, thus supporting the notion that those higher in communion should perceive more of the items listed to constitute acts of infidelity. It was also predicted that unmitigated agency would be negatively associated with the number of items endorsed, and this was also the case, $r(360)=-0.15, P<0.004$. Finally, it was predicted that agency would show no association with perceptions of infidelity, and this was also supported, $r(361)=-0.03$, $P=0.570$.

\section{Ancillary analyses}

Given that the infidelity questionnaire used in the current study was new, a factor analysis was carried out in order to examine subtleties in the patterns of responses. A principal components analysis using varimax rotation was computed, showing evidence of five factors within the questionnaire. These five factors were sexual activity, suggestivity, fantasy, trust, and other commitment. See Appendix A for a breakdown of which items loaded on which factor.

The originally reported sex difference in which females perceived more items to constitute infidelity held for four of the five subtypes, with the fantasy factor being the sole exception (although even in this case the means were in the original direction).

When examining the pattern of correlations between these five factors and the gender-related traits, some interesting patterns emerge. See Table 2 for the specific correlations among the variables as well as $\alpha$ reliabilities. Unmitigated communion was positively associated with the fantasy, trust, and other commitment factors, suggesting that these specific types of infidelity were responsible for the originally reported positive correlation between unmitigated communion and overall perceptions of infidelity. Communion was positively correlated with all five subtypes of infidelity perceptions, and agency remained unrelated to any of the five subtypes of infidelity. Both of these patterns support the originally reported associations with infidelity perceptions as a whole. Finally, unmitigated agency was negatively associated with the fantasy and other commitment factors, suggesting that these two specific subtypes of infidelity were responsible for the originally reported negative association between unmitigated agency and infidelity perceptions. 
Table 2 Correlations and $\alpha$ for the main study variables

\begin{tabular}{|c|c|c|c|c|c|c|c|c|c|}
\hline & $\mathbf{I}$ & 2 & 3 & 4 & 5 & 6 & 7 & 8 & 9 \\
\hline I. Communion & $(0.76)$ & $0.12^{*}$ & $0.46 * * *$ & $-0.37 * * *$ & $0.13^{*}$ & $0.14 * *$ & $0.13 *$ & $0.12^{*}$ & $0.11 *$ \\
\hline 2. Agency & & $(0.76)$ & $-0.21 * * *$ & 0.09 & 0.01 & -0.02 & -0.03 & 0.01 & -0.09 \\
\hline 3. Unmitigated communion & & & $(0.74)$ & $-0.22 * * *$ & 0.06 & 0.07 & $0.21^{* * *}$ & $0.11 *$ & $0.12 *$ \\
\hline 4. Unmitigated agency & & & & $(0.72)$ & -0.04 & -0.07 & $-0.18 * * *$ & -0.04 & $-0.19 * * *$ \\
\hline 5. Sexual activity & & & & & $(0.86)$ & $0.19 * * *$ & 0.09 & 0.03 & $0.14 * * *$ \\
\hline 6. Suggestivity & & & & & & $(0.66)$ & $0.35 * * *$ & $0.27^{* * *}$ & $0.28 * * *$ \\
\hline 7. Fantasy & & & & & & & $(0.67)$ & $0.23^{* * *}$ & $0.22 * * *$ \\
\hline 8. Trust & & & & & & & & $(0.69)$ & $0.13^{*}$ \\
\hline 9. Other commitment & & & & & & & & & $(0.50)$ \\
\hline
\end{tabular}

Notes: $* p<0.05 ; * * p<0.01 ; * * * p<0.001$.

\section{Discussion}

The present study provides support for all hypotheses laid out in the introduction. First, female participants checked off more items on the perceived infidelity questionnaire than did males. This difference was hypothesized because previous research has shown that females have a stronger sensitivity toward infidelity than do males, particularly perceived emotional infidelity. ${ }^{6}$ Evolutionary psychologists have devoted significant attention to the question of gender differences in emotional responses to betrayal in romantic relationships. ${ }^{10-15}$ Evolutionary psychologists believe that because of paternity uncertainty, males should become more jealous over their partners' sexual infidelity than emotional infidelity. This is because sexual infidelity presents the possibility of the evolutionary cost of devoting resources to the offspring of another male. In contrast, females are certain about their genetic link to their offspring but face the threat that their mates will withdraw resources from their offspring, and mates' attachment to another female is likely to lead to diversion of resources to that female and her offspring. Thus, the theory suggests that females are more likely to become upset by signs of resource withdrawal (foreshadowed by emotional infidelity) by their mates than by signs of perceived sexual infidelity. This theory is the currently accepted wisdom about evolved responses to infidelity and best explains why there are gender differences in response to perceptions of infidelity. Research on evolutionary responses to betrayal and other previous research has looked at what happens once an individual perceives infidelity and why they perceive it. Our findings suggest that those perceptions will vary based on personality traits and biological sex. The current study has sought to identify what those perceptions of infidelity are before they are acted on and begin to promote jealousy and anger in a relationship.

Second, females scored higher on communion and unmitigated communion, whereas males scored higher on agency and unmitigated agency. ${ }^{7}$ These patterns are all also in line with previous research.

For the gender-related traits, there were multiple parts to the hypothesis. We first hypothesized a positive correlation between unmitigated communion and infidelity perceptions. The results support this hypothesis. A potential reason for this significant correlation between unmitigated communion and infidelity perceptions is primarily the type of personality traits that unmitigated communion individuals display. These personality traits include low self-esteem, a more codependent or dependent nature, and being overly concerned or obsessive with the state of a relationship or with the partner involved. ${ }^{9}$ As a result, these individuals might have perceived more of the items on the infidelity questionnaire as being a threat to their relationships in order to protect these relationships. This effect would be similar to being oversensitive to potential harm from the environment. If problems are detected early, there is a better chance of intervention to preserve the relationship.

We next hypothesized a negative correlation between unmitigated agency and infidelity perceptions. The results also support this hypothesis. Unmitigated agency is said to be correlated with traits such as hostility, greed, and arrogance and with being overly concerned with oneself. ${ }^{9}$ Because of these traits, we believe a potential reason why individuals scoring high on this trait checked off fewer items on the infidelity questionnaire is because unmitigated agency individuals simply lack concern for others or perceived threats to relationships, due in part to their self-absorption to the exclusion of others.

Third, we hypothesized that there would be no correlation between agency and perceived infidelity as a result of the emotional security of individuals scoring high in agency, and this hypothesis was supported. Agency individuals are characteristically confident, and as a result we expected no extreme number of items checked or unchecked on the 
infidelity questionnaire, as their views of infidelity are more than likely more realistic than the other gender-related trait perceptions. However, agency individuals' lack of perceptions of infidelity could be dangerous, in that they are not expecting their partner to commit infidelity and therefore set themselves up for a potentially unexpected and hurtful experience.

Finally, we examined the correlation between communion and infidelity perceptions. We were unsure of the relationship that communion would have with these perceptions. As the results indicate, communion was positively correlated with infidelity perceptions. A potential cause for the positive correlation could be that those scoring high on communion are overinvolved or overindulgent in their relationships, much like the obsessiveness of unmitigated communion individuals. It is also possible that communion serves a priming factor for relationships that makes individuals high in this trait more sensitive to both the positive and negative aspects of their relationships. This would explain why communion is associated with both supportive interactions (as demonstrated by past research) and perceptions of infidelity (as demonstrated in this study).

It appears that the patterns of associations discussed so far hold not only for the overall perceptions of infidelity using the entire infidelity questionnaire but also for the subtypes of infidelity derived from a factor analysis of the larger instrument. There are two notable points to make here. First, unmitigated communion's association with infidelity was driven by the fantasy, trust, and other commitment factors rather than the sexual activity and suggestivity factors, which suggests that someone high in unmitigated communion might be predisposed to view breaches in more emotional, as opposed to physical, aspects of relationships as constituting infidelity. Second, communion was the only trait to be associated with either sexual activity or suggestivity, hinting at the notion that communion is more closely tied to these aspects of infidelity than the other traits. This difference in the patterns of relationships between communion and unmitigated communion and the gender-related traits could well be highlighting the differences between a healthy and an unhealthy focus on relationships and what constitutes breaches of fidelity within these relationships.

Now that these associations between the traits and infidelity perceptions have been revealed, there are a few potential real-world applications to consider. In terms of the gender-related traits, there are a few combinations of personality types that could result in very negative outcomes. First, the combination of two individuals scoring high on unmitigated communion could prove to be a detrimental combination of personality styles. For one, individuals high in unmitigated communion tend to be obsessive and overinvolved in their relationship. If both people in the relationship felt that way, there could potentially be constant anxiety and worry about the nature of their partnership. Also, because they maintain a constant concern for their relationship, there would be no complementary side of the relationship to put the other individual "in check" or to bring them to a realistic, trusting, and relaxed state of mind. In fact, for these two individuals, the anxiety would be expected to double. As a result, the relationship between two unmitigated communion individuals would show to be an unhealthy relationship for both involved.

On the other hand, a relationship between two individuals scoring high in levels of agency might prove to be a very positive companionship. This is because those high in agency are individuals considered to be confident and more self-focused, but not to the exclusion of others. These two individuals should display security in their relationship, and as a result be able to focus on themselves in a healthy way while still maintaining a focus on their partnership (unlike the unmitigated communion couple, in which the focus is primarily on their partner and on their relationship, exclusive of themselves). These combinations of personality traits would be important to take into consideration before becoming involved with an individual whose personality characteristics may clash.

Further research can be done in evaluating more variables, such as age, type of relationship the individual is involved in, and sexual orientation. Other components, such as subgroups of infidelity (emotional infidelity and sexual infidelity), could be analyzed in detail to see which, in general, is more universally distressing and to whom. The gender-related traits and the ways in which they influence perceptions of infidelity can also be examined in further detail. Most importantly, this research could potentially be extended to include actual behaviors rather than asking participants to consider "what if" situations.

Potential flaws in this study are the sample of college students who participated in the questionnaire. The students were a convenience sample, making it hard to generalize to the rest of the population. Also, college students are a unique sample of individuals, and their views on sex and infidelity are potentially equally unique. As a result, these views could have skewed the ability to generalize to the population. Were this study to be conducted again, a different sample of individuals would be used, specifically a sample with a broader age range, and not consisting entirely of college students. This would be done in order to show perceptions of all types 
of individuals, not just college students, making the results generalizable to the wider population.

Another inevitable limitation of the study is social desirability. The questionnaires were administered to large classrooms of students, and because the surveys contained visibly personal information, many students may not have felt comfortable being completely honest in selecting the items they did or did not consider infidelity. For example, if an individual had a high tolerance for concepts of infidelity but thought that it would look improper to select fewer items than the average individual, they may have selected more items on the infidelity questionnaire to compensate. It is also possible that the opposite effect could be present, in that participants may have selected fewer items than appropriate in order to not appear too "open-minded" when it comes to perceptions of infidelity. This is a limitation that must be considered in deciphering the honesty of the participants.

A third potential flaw in the study is that the questionnaire used to assess perceptions of infidelity is not a well-validated instrument, although it does correlate in meaningful ways with pre-existing well-validated measures of other constructs. Future research should seek to provide further support for the validity and reliability of this instrument. Finally, it should be noted that the magnitude of the associations reported in this paper is small; however, it is believed that these relationships can hold some practical and clinical significance, as discussed previously.

It is obvious that the implications of infidelity can have many effects on an individual and their relationships. It is evident from the results of the current study that perceptions of infidelity can result not only from differences in gender but also from differences in personality traits. The success of this study was in finding that there is a strong connection between perceptions of infidelity and the gender-related traits. This study will serve in furthering the available research about infidelity, as well as the available research about the genderrelated traits. As is obvious in any relationship, infidelity can hurt and shatter the trust in a relationship. However, if individuals are aware of their partner's perceptions of infidelity, it may serve them in providing less heartache and also contribute to a more understanding and healthy relationship for both individuals involved.

\section{Disclosure}

The authors report no conflicts of interest in this work.

\section{References}

1. Chuick C. Gender and Infidelity: a Study of the Relationship Between Conformity to Masculine Norms and Extrarelational Involvement [dissertation]. Iowa City, IA: University of Iowa; 2009.

2. Kinsey AC, Pomeroy WB, Martin CE. Sexual Behavior in the Human Male. Philadelphia, PA: Saunders; 1948.

3. Whitty MT, Quigley L. Emotional and sexual infidelity offline and in cyberspace. J Marital Fam Ther. 2008;34(4):461-468.

4. Blow AJ, Hartnett K. Infidelity in committed relationships. II: A substantive review. J Marital Fam Ther. 2005;31(2):217-233.

5. Miller SL, Maner JK. Sex differences in response to sexual versus emotional infidelity: the moderating role of individual differences. Pers Indiv Differ. 2009;46(3):287-291.

6. Shackelford TK, Buss DM. Betrayal in mateships, friendships, and coalitions. Pers Soc Psychol Bull. 1996;22:1151-1164.

7. Helgeson VS. Relation of agency and communion to well-being: evidence and potential explanations. Psychol Bull. 1994;116:412-428.

8. Bakan D. The Duality of Human Existence. Chicago, IL: Rand McNally; 1966.

9. Helgeson VS, Fritz HL. Unmitigated agency and unmitigated communion: distinctions from agency and communion. $J$ Res Pers. 1999;33:131-158.

10. Spence JT, Helmreich RL. The many faces of androgyny: A reply to Locksley and Colten. J Pers Soc Psychol. 1979;37:1032-1046.

11. Spence JT, Helmreich RL, Holahan CK. Negative and positive components of psychological masculinity and femininity and their relationships to self-reports of neurotic and acting out behaviors. J Pers Soc Psychol. 1979;37:1673-1682.

12. Fritz HL, Helgeson VS. Distinctions of unmitigated communion from communion: Self-neglect and overinvolvement with others. J Pers Soc Psychol. 1998;75:121-140.

13. Helgeson VS. The role of masculinity in a prognostic predictor of heart attack severity. Sex roles. 1990;22:755-774.

14. Buss DM, Larsen RJ, Westen D, Semmelroth J. Sex differences in jealousy: evolution, physiology, and psychology. Psychol Sci. 1992;3: 251-255.

15. Buss DM. The Dangerous Passion: Why Jealousy is as Necessary as Love and Sex. New York, NY: Free Press; 2000.

16. DeSteno DA, Salovey P. Evolutionary origins of sex differences in jealousy? Questioning the 'fitness' of the model. Psychol Sci. 1992;7: 367-372.

17. Harris CR, Christenfeld N. Gender, jealousy, and reason. Psychol Sci. 1996;7:364-366

18. Sabini J, Green MC. Emotional responses to sexual and emotional infidelity: constants and differences across genders, samples, and methods. Pers Soc Psychol Bull. 2004;30:1375-1388.

19. Sagarin BJ. Reconsidering evolved sex differences in jealousy: comment on Harris (2003). Pers Soc Psychol Rev. 2005;9:62-75. 


\section{Appendix A - Infidelity questionnaire divided by factor Other commitment}

1. Being in a committed, monogamous relationship while hiding an emotional attachment to someone other than your partner

2. Being in a committed, monogamous relationship while hiding a physical attachment to someone other than your partner

\section{Sexual activity}

1. An intimate kiss (kiss on the lips) with someone other than your partner

2. Engaging in sexual intercourse with someone other than your partner

3. Oral sex with someone other than your partner

4. A sexual phone conversation with someone other than your partner

5. Sexting (the act of sending sexually explicit messages or photos electronically, usually between cell phones)

\section{Fantasy}

1. Fantasizing about someone other than your partner

2. Viewing pornographic material

3. Masturbation

4. Going to a strip club without your partner

\section{Suggestivity}

1. A casual kiss (kiss on the cheek) with someone other than your partner

2. Sharing an intimate emotional bond with someone other than your partner

3. Flirting with someone other than your partner

4. Going out for lunch or drinks (a "date") with someone other than your partner

5. An emotional and intimate phone conversation with someone other than your partner

6. Any kind of physical contact with someone other than your partner

\section{Trust}

1. General dishonesty (lying about anything to your partner)

2. Any breach of trust in the relationship
Psychology Research and Behavior Management

\section{Publish your work in this journal}

Psychology Research and Behavior Management is an international, peerreviewed, open access journal focusing on the science of psychology and its application in behavior management to develop improved outcomes in the clinical, educational, sports and business arenas. Specific topics covered include: Neuroscience, memory \& decision making; Behavior

\section{Dovepress}

modification \& management; Clinical applications; Business \& sports performance management; Social and developmental studies; Animal studies. The manuscript management system is completely online and includes a quick and fair peer-review system. Visit http://www.dovepress. com/testimonials.php to read real quotes from published authors. 